\title{
Building your Babylon
}

\author{
Ron Bridges $\mathrm{MD}^{1}$, Paul C Adams MD FRCPC ${ }^{2}$, Editor-in-Chief
}

$\mathrm{R}_{\mathrm{f}}^{\mathrm{o}}$

on Bridges is the Head of the Division of Gastroenterology Professor of Medicine at the University of Calgary, Calgary, Alberta. He has been actively involved in the development of the Forzani and MacPhail Colon Cancer Screening Centre (CCSC) at the University of Calgary, and has been the Medical Director for the centre during the formative planning and implementation stages. PA: Many gastroenterologists would like to expand into ambulatory endoscopy and yet there are many financial, political or regulatory hurdles. You have successfully built a large ambulatory endoscopy centre at the University of Calgary. Can you outline the history of the project?

RB: The CHR recognized that there was inadequate hospital capacity to manage present and future demand for gastroenterology procedures due to population growth, increased demand for endoscopic therapeutic techniques and a change in the standard of care related to colon cancer screening. Recognizing this, and with an objective for effective utilization of the incoming resources, a competitive process conducted

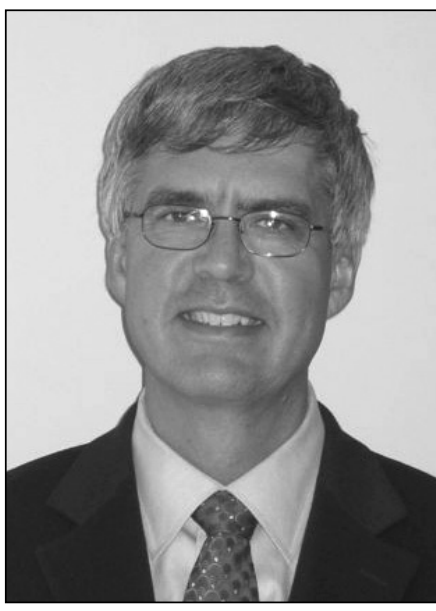

Ron Bridges is the Head, Division of Gastroenterology for the Calgary Health Region and Clinical Professon of Medicine at the University of Calgary, Calgary, Alberta
Public Benefits document. The University of Calgary agreed to provide space in the new Teaching Research and Wellness facility at the Foothills Medical Centre site. Community support was obtained from The Forzani Group Foundation and $\mathrm{Mr}$ Keith MacPhail. They generously donated \$2.7 million to purchase endoscopic and other related medical equipment necessary to operationalize the CCSC, and have committed to play an important role in public awareness and education programs at the Centre. Financial support in the order of \$1 million has been acquired from the Alberta Cancer Board to enable the development of a comprehensive electronic medical record system that will facilitate patient care, reporting, quality care initiatives and clinical research protocols. The CCSC is collaborating with the provincial colon cancer screening program led by the Alberta Cancer Board.

PA: How did you convince your regional health authority of the need for this facility?

RB: Wait time data collected by the Canadian Association of Gastroenterology demonstrated that Alberta and the Calgary region had among the longest wait times in Canada to access specialty care in gastroenterology. This, in combiby the CHR in 2003 resulted in the University of Calgary's Division of Gastroenterology becoming the selected proponent to deliver specific endoscopic colon cancer screening and surveillance procedures to the region's stable outpatient population. Since then, negotiations have been held and a 10-year agreement was reached with the CHR to provide a competitive fixed procedural fee-for-services at the CCSC. The arrangement has several objectives, all aiming to reduce the incidence of colon cancer, with resulting improved quality of life to people and decreased costs to the CHR. A truly collaborative program involving the CHR, the University of Calgary, the Calgary community and the Alberta Cancer Board has been developed. This initiative ensures that the provision of contracted services strictly adheres to the principles of the Canada Health Act and provincial legislative requirements, including the Health Professions Act and the Health Information Act. Many individuals have contributed in a significant manner and several hurdles have been cleared in the process of implementing the CCSC. Outpatient ambulatory surgical facilities, including endoscopy centres, are regulated by the College of Physicians and Surgeons of Alberta. The CCSC must complete a detailed accreditation process that addresses all aspects of operation before opening. Ministerial approval from the Government of Alberta was obtained following the submission of a detailed Net nation with a rapidly growing regional population, necessitated the development of a plan to provide additional endoscopy resources for the citizens of Calgary. We are fortunate to have an administration that is supportive of novel programs in the advancement of health care. The program has resulted in the development of a plan for sustainable contractual arrangements that outline all elements of the business arrangement and are based on a foundation of mutually understood expectations, goals and performance outcomes. The partnership contributes to the standardization of treatment and related processes between public and private facilities in the CHR at a known and sustainable cost. The CCSC has enhanced the recruitment of physicians and enabled trials to utilize people in novel roles to facilitate an efficiently operated program. Once these roles are demonstrated to be effective, they will be employed at CHR hospital endoscopy units and clinics. By employing aggressive strategies to recruit gastroenterologists, the CHR expects physician capacity to increase, making it possible to deliver more timely care and reduce the size of waiting lists. By moving current screening and surveillance procedures from the acute care system, additional capacity will be created for the assessment of people with acute and chronic gastrointestinal diseases. In addition to providing high-quality clinical care, the CCSC will

${ }^{1}$ Division of Gastroenterology, University of Calgary, Calgary, Alberta; ${ }^{2}$ University Hospital, London, Ontario

Correspondence: Dr Ron Bridges, Division of Gastroenterology, 3330 Hospital Drive Northwest, Calgary, Alberta T2N 4N1.

Telephone 403-220-4245, fax 403-283-5594, e-mail bridges@ucalgary.ca

Received and accepted for publication October 31, 2007 


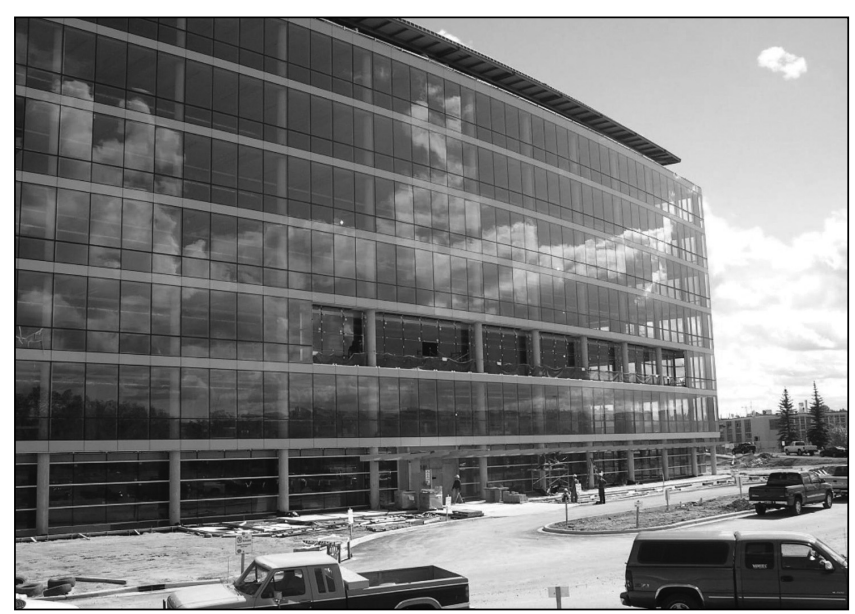

The exterior of the new Teaching Research and Wellness facility at the University of Calgary's Foothills Medical Centre site (Calgary, Alberta). The Forzani and MacPhail Colon Cancer Screening Centre is located on the sixth floor

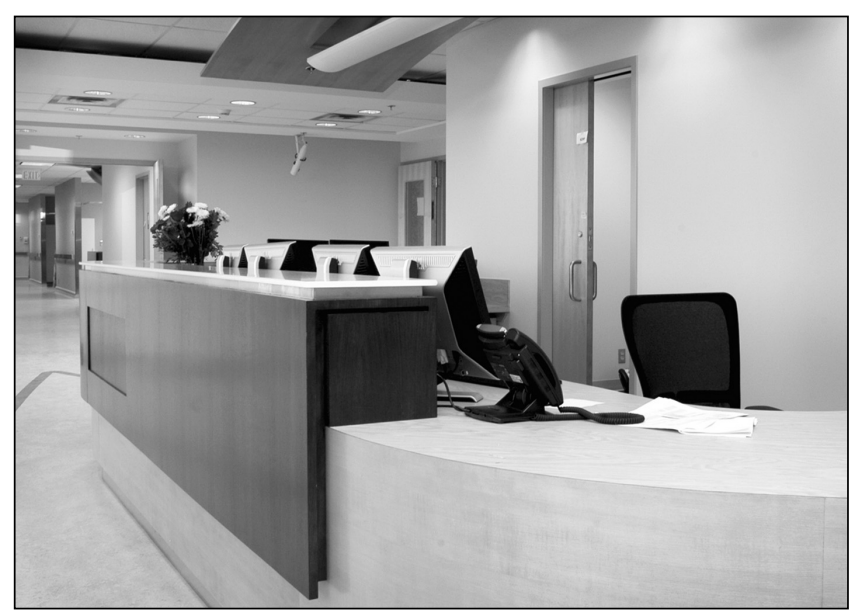

The reception area of the Forzani and MacPhail Colon Cancer Screening Centre

evaluate existing and novel colon cancer screening techniques from both an efficacy and cost-effective perspective.

PA: Can you outline the layout of the clinic?

RB: The CCSC is located on the sixth floor of the new Teaching, Research and Wellness facility at the University of Calgary's Foothills Medical Centre site. It occupies 23,000 square feet and consists of six endoscopy rooms, three reprocessing rooms, 24 recovery beds, six nurse clinician preassessment rooms, research, business and biomedical offices, a theatre for public and professional education activities, meeting rooms and waiting room educational modules. The CCSC has a fully integrated electronic medical record system, including endoscopic and nursing records. Forty-two physicians working at the CCSC are members of the CHR's Division of Gastroenterology, while six physicians belong to the Division of Colorectal Surgery. In the first year of operation, 10,000 colonoscopies will be completed and by the third year of operation, we expect capacity to increase to 20,000 colonoscopies annually. The focus of the CCSC will be on the provision of colonoscopy related to screening for, and the

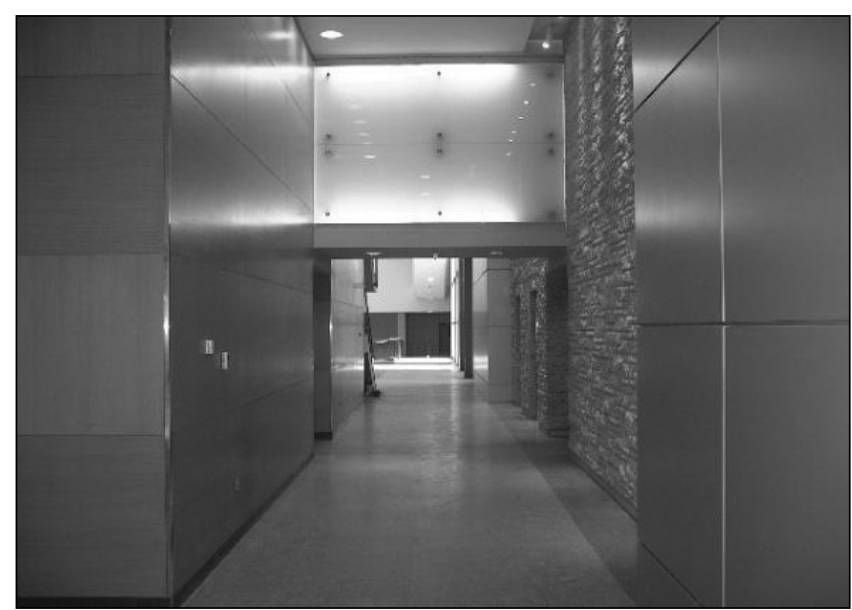

A hallway of the Forzani and MacPhail Colon Cancer Screening Centre

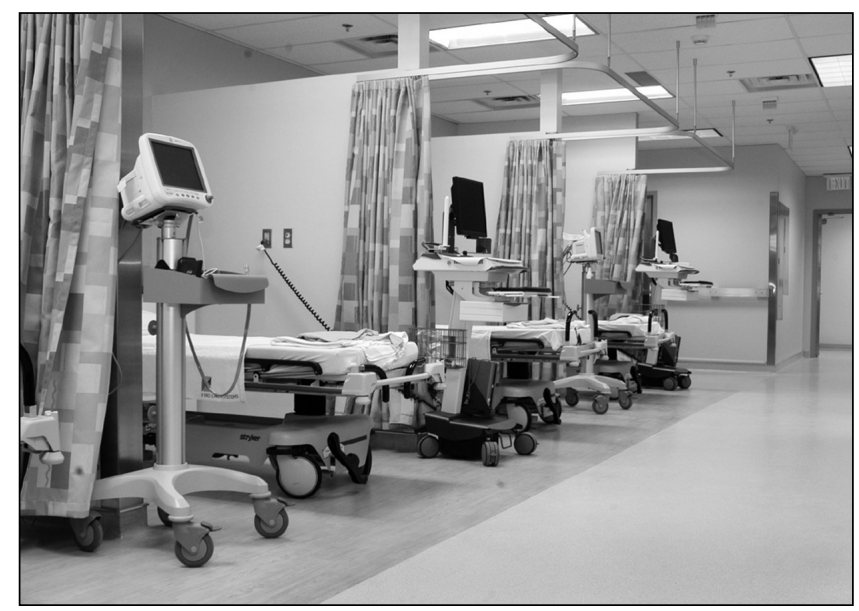

The recovery room in the Forzani and MacPhail Colon Cancer Screening Centre

surveillance of, colon cancer. Physician fees are paid by Alberta Health and Wellness at rates defined by the current provincial fee code. In the same facility, a computed tomography scanner installed with donor funds will allow for the provision of large research protocols related to virtual colonography. A biorepository will be established, allowing for the collection of tissue, urine, stool and serum samples from a large population of people undergoing colon cancer screening. This enhances the development of a multidisciplinary network of biomedical, clinical, health services and population health researchers to assess the efficacy of various screening techniques and also provide a large bank of normal controls for other gastrointestinal research projects in the division.

PA: Are most of your endoscopists on fee-for-service arrangements or salary?

RB: Physicians working at the CCSC are almost equally divided between fee-for-service and the Department of Medicine's Alternate Relationship Plan. None of the participating physicians are on salary. For those physicians in the Alternate Relationship Plan, their services at the CCSC are a 
component of clinical activities described in their individual service agreement.

PA: Does this clinic generate a profit?

RB: The University of Calgary's Faculty of Medicine and the Division of Gastroenterology receive a fee for operating the clinic. In accordance with contractual agreements, the funds are fully reinvested in public education, innovation and research related to colon cancer screening, prevention and therapy. In this business model, no profits are directed to physician or business accounts.

PA: How would you contrast your clinic with ambulatory endoscopy clinics in which the doctors pay overhead fees to a business partner?

RB: The Forzani and MacPhail CCSC is a truly collaborative effort involving the CHR, the University of Calgary's Division of Gastroenterology and the Calgary community. There is a focused commitment on the provision of colon cancer screening in an organized, standardized and accountable manner to the citizens of the Calgary region. In this model, all parties are engaged, and funding for the operation of the Centre does not rest on the shoulders of physicians working at the Centre. Unlike other ambulatory endoscopy clinics, the CCSC has organized educational and research components included in its operational goals. In collaboration with Dr Roland Valori, a comprehensive quality assurance program has been developed to follow all aspects of care delivered at the Centre.

PA: Are there any threats to the future viability of these types of clinics (virtual colonoscopy, other advanced imaging, government regulations)?

RB: It is difficult to predict the future of all aspects of medical care, including colon cancer screening, in the Canadian health care system. With an increasing focus on wellness and prevention, I believe there is significant opportunity for this type of collaborative venture. The CCSC will be actively engaged in the evaluation of all current and potential techniques for colon cancer screening. If a new screening technique is determined to be more effective and cost-efficient, it will be adopted for use at the CCSC. With continued population growth and an aging regional population, there will be continued demand for endoscopic services in the volumes outlined for the CCSC for many years to come. In the future, the endoscopic capacity at the CCSC may well be increasingly focused on the removal of polyps identified through another screening modality. 


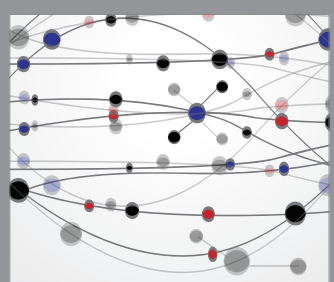

The Scientific World Journal
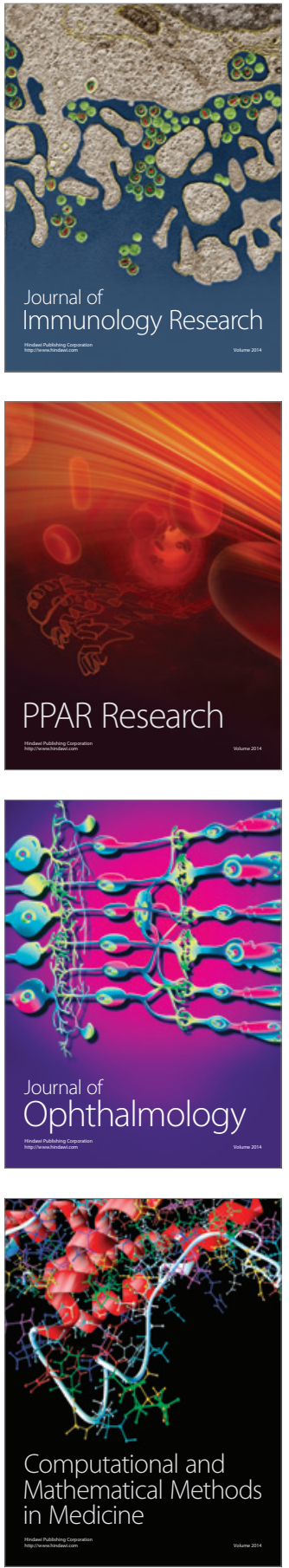

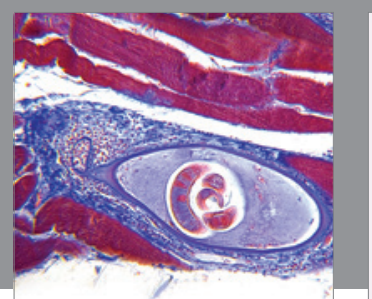

Gastroenterology Research and Practice

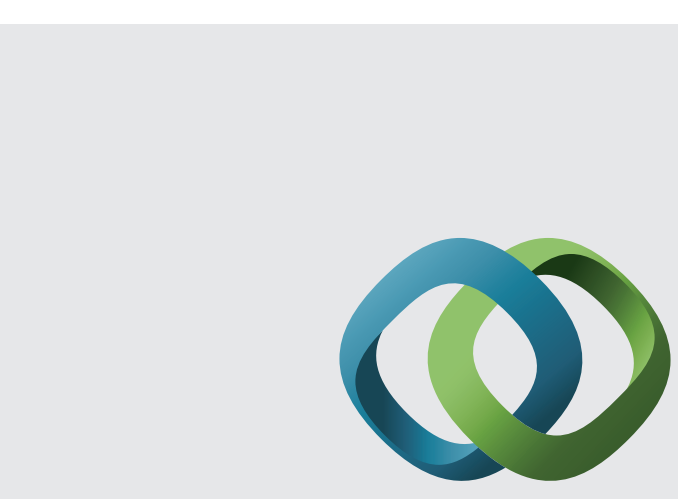

\section{Hindawi}

Submit your manuscripts at

http://www.hindawi.com
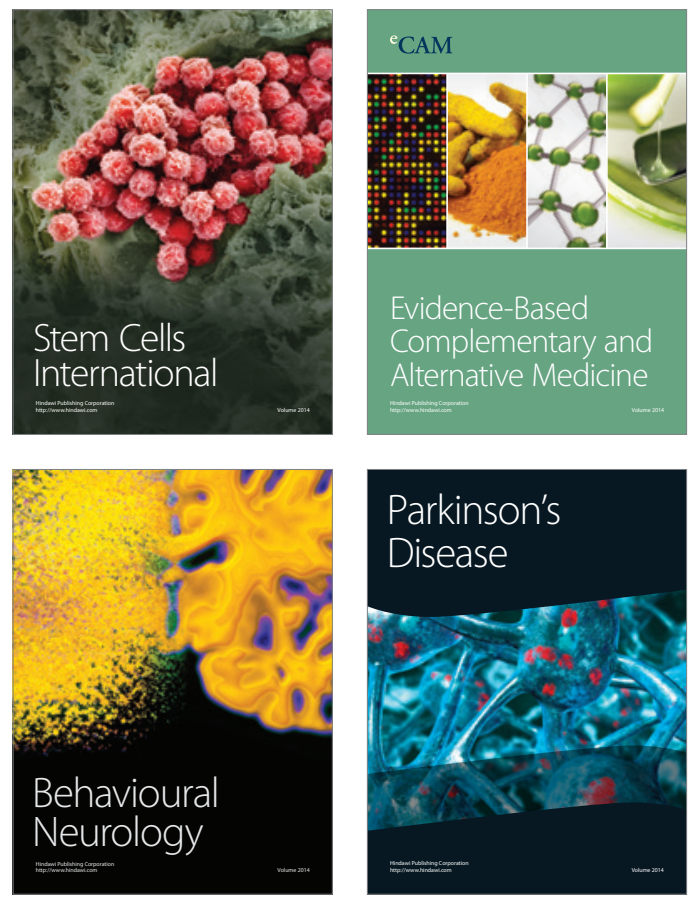
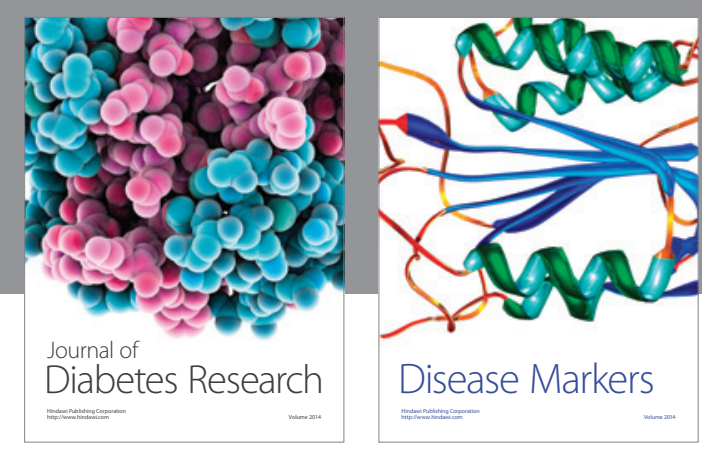

Disease Markers
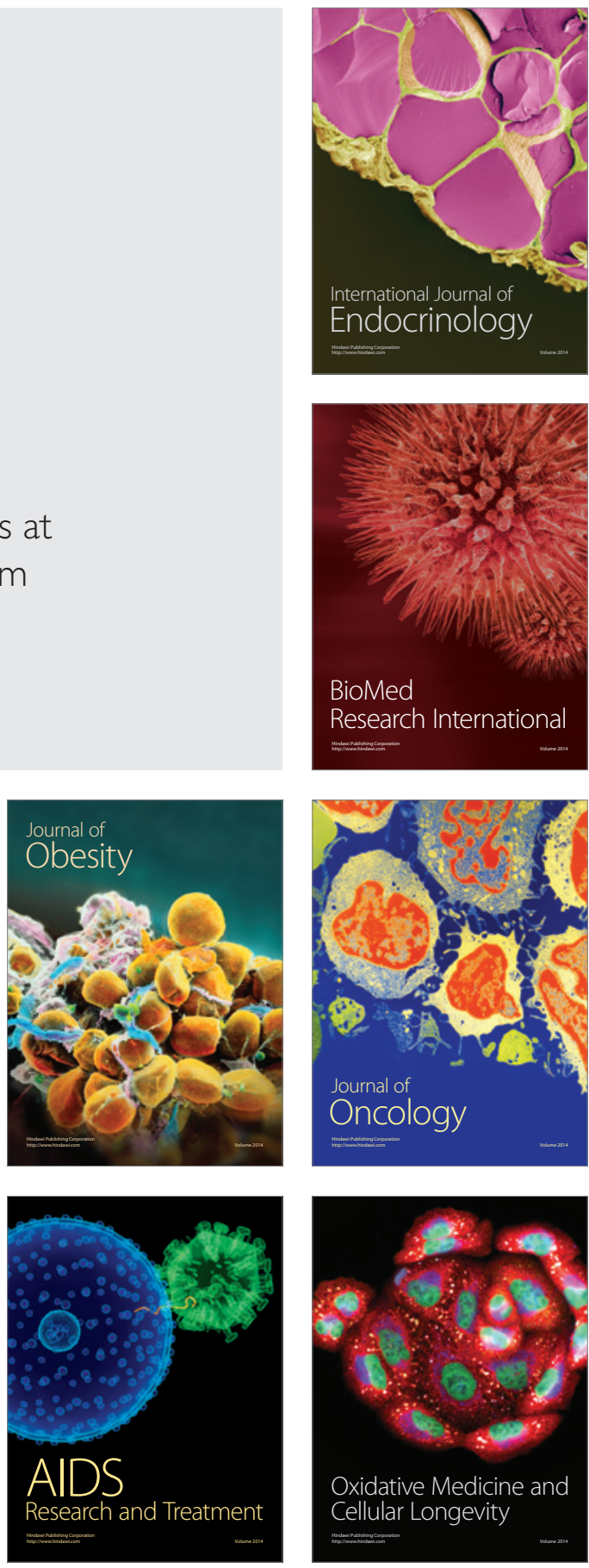